\title{
Basic science: (May 2008)
}

1. Al-Azawi D, Mc llroy M, Kelly G, Redmond AM, Bane FT, Cocchiglia S, Hill ADK, Young LS. Ets-2 and p160 proteins collaborate to regulate c-Myc in endocrine resistant breast cancer. Oncogene 2008; 27: 3021-3031.

2. Barabutis N, Schally AV. Knocking down gene expression for growth hormone-releasing hormone inhibits proliferation of human cancer cell lines. Br J Cancer 2008; 98: 1790-1796.

3. Boulay A, Breuleux M, Stephan C, Fux C, Brisken $C$, Fiche $M$, Wartmann $M$, Stumm $M$, Lane HA, Hynes NE. The Ret receptor tyrosine kinase pathway functionally interacts with the ER $\alpha$ pathway in breast cancer. Cancer Res 2008; 68: 3743-3751.

4. Elsheikh S, Green AR, Aleskandarany MA, Grainge M, Paish CE, Lambros MBK, Reis JS, Ellis IO. CCND1 amplification and cyclin D1 expression in breast cancer and their relation with proteomic subgroups and patient outcome. Breast Cancer Res Treat 2008; 109: 325-335.

5. Euhus DM, Bu DW, Milchgrub S, Xie XJ, Bian A, Leitch AM, Lewis CM. DNA methylation in benign breast epithelium in relation to age and breast cancer risk. Cancer Epidemiol Biomarkers Prev 2008; 17: 1051-1059.

6. Farrugia DJ, Agarwal MK, Pankratz VS, Deffenbaugh AM, Pruss D, Frye C, Wadum L, Johnson K, Mentlick J, Tavtigian SV, Goldgar $D E$, Couch FJ. Functional assays for classification of BRCA2 variants of uncertain significance. Cancer Res 2008; 68: 3523-3531.

7. Fereshteh MP, Tilli MT, Kim SE, Xu JM, O'Malley BW, Wellstein A, Furth PA, Riegel AT. The nuclear receptor coactivator amplified in breast cancer-1 is required for neu (ErbB2/ HER2) activation, signaling, and mammary tumorigenesis in mice. Cancer Res 2008; 68: 3697-3706.

8. Finak G, Bertos N, Pepin F, Sadekova S, Souleimanova M, Zhao H, Chen HY, Omeroglu G, Meterissian S, Omeroglu A, Hallett M, Park M. Stromal gene expression predicts clinical

$\mathrm{BCO} / 842 / 2008 / \mathrm{JW}$ outcome in breast cancer. Nat Med 2008; 14: 518-527.

9. Frech MS, Torre KM, Robinson GW, Furth PA. Loss of cyclin D1 in concert with deregulated estrogen receptor $\alpha$ expression induces DNA damage response activation and interrupts mammary gland morphogenesis. Oncogene 2008; 27: 3186-3193.

10. Golipour A, Myers D, Seagroves T, Murphy D, Evan GI, Donoghue DJ, Moorehead RA, Porter LA. The Spy1/RINGO family represents a novel mechanism regulating mammary growth and tumorigenesis. Cancer Res 2008; 68: 3591-3600.

11. Hodges-Gallagher L, Valentine CD, El Bader S, Kushner PJ. Estrogen receptor $\beta$ increases the efficacy of antiestrogens by effects on apoptosis and cell cycling in breast cancer cells. Breast Cancer Res Treat 2008; 109: 241-250.

12. Hollingshead BD, Beischlag TV, DiNatale BC, Ramadoss P, Perdew GH. Inflammatory signaling and aryl hydrocarbon receptor mediate synergistic induction of interleukin 6 in MCF-7 cells. Cancer Res 2008; 68: 3609-3617.

13. Howarth KD, Blood KA, Ng BL, Beavis JC, Chua Y, Cooke SL, Raby S, Ichimura K, Collins VP, Carter NP, Edwards PAW. Array painting reveals a high frequency of balanced translocations in breast cancer cell lines that break in cancer-relevant genes. Oncogene 2008; 27: 3345-3359.

14. Hu M, Yao J, Carroll DK, Weremowicz S, Chen $H$, Carrasco D, Richardson A, Violette S, Nikolskaya T, Nikolsky Y, Bauerlein EL, Hahn WC, Gelman RS, Allred C, Bissell MJ, Schnitt $\mathrm{S}$, Polyak K. Regulation of in situ to invasive breast carcinoma transition. Cancer Cell 2008; 13: 394-406.

15. Jiao W, Lin HM, Datta J, Braunschweig T, Chung JY, Hewitt SM, Rane SG. Aberrant nucleocytoplasmic localization of the retinoblastoma tumor suppressor protein in human cancer correlates with moderate/poor tumor differentiation. Oncogene 2008; 27: 3156-3164.

16. Kaklamani VG, Sadim M, Hsi A, Offit K, Oddoux C, Ostrer H, Ahsan H, Pasche B, Mantzoros C. 
Variants of the adiponectin and adiponectin receptor 1 genes and breast cancer risk. Cancer Res 2008; 68: 3178-3184.

17. Kumar AS, Benz CC, Shim V, Minami CA, Moore DH, Esserman LJ. Estrogen receptornegative breast cancer is less likely to arise among lipophilic statin users. Cancer Epidemiol Biomarkers Prev 2008; 17: 1028-1033.

18. Li XX, Lewis MT, Huang J, Gutierrez C, Osborne CK, Wu MF, Hilsenbeck SG, Pavlick A, Zhang XM, Chamness GC, Wong $\mathrm{H}$, Rosen $\mathrm{J}$, Chang JC. Intrinsic resistance of tumorigenic breast cancer cells to chemotherapy. J Natl Cancer Inst 2008; 100: 672-679.

19. Ma XJ, Salunga R, Dahiya S, Wang W, Carney E, Durbecq V, Harris A, Goss P, Sotiriou C, Erlander $M$, Sgroi D. A five-gene molecular grade index and HOXB13: IL17BR are complementary prognostic factors in early stage breast cancer. Clin Cancer Res 2008; 14: 2601-2608.

20. Mao C, Patterson NM, Cherian MT, Aninye IO, Zhang C, Montoya JB, Cheng J, Putt KS, Hergenrother PJ, Wilson EM, Nardulli AM, Nordeen SK, Shapiro DJ. A new small molecule inhibitor of estrogen receptor $\alpha$ binding to estrogen response elements blocks estrogendependent growth of cancer cells. $\mathrm{J}$ Biol Chem 2008; 283: 12819-12830.

21. Melchor L, Honrado E, Garcia MJ, Alvarez S, Palacios J, Osorio A, Nathanson KL, Benitez J. Distinct genomic aberration patterns are found in familial breast cancer associated with different immunohistochemical subtypes. Oncogene 2008; 27: 3165-3175.

22. Mueller KL, Hunter LA, Ethier SP, Boerner JL. Met and C-Src cooperate to compensate for loss of epidermal growth factor receptor kinase activity in breast cancer cells. Cancer Res 2008; 68: 3314-3322.

23. Northey JJ, Chmielecki J, Ngan E, Russo C, Annis MG, Muller WJ, Siegel PM. Signaling through ShcA is required for transforming growth factor $\beta$ - and Neu/ErbB-2-induced breast cancer cell motility and invasion. $\mathrm{Mol}$ Cell Biol 2008; 28: 3162-3176.

24. Qiu W, Hu M, Sridhar A, Opeskin K, Fox S, Shipitsin M, Trivett M, Thompson ER, Ramakrishna M, Gorringe KL, Polyak K, Haviv I, Campbell IG. No evidence of clonal somatic genetic alterations in cancer-associated fibroblasts from human breast and ovarian carcinomas. Nat Genet 2008; 40: 650-655.
25. Schug TT, Berry DC, Toshkov IA, Cheng L, Nikitin AY, Noy N. Overcoming retinoic acidresistance of mammary carcinomas by diverting retinoic acid from PPAR $\beta / \delta$ to RAR. Proc Natl Acad Sci USA 2008; 105: 7546-7551.

26. Shafee N, Smith CR, Wei SZ, Kim Y, Mills GB, Hortobagyi GN, Stanbridge EJ, Lee E. Cancer stem cells contribute to cisplatin resistance in Brca1/p53-mediated mouse mammary tumors. Cancer Res 2008; 68: 3243-3250.

27. Shakya R, Szabolcs M, McCarthy E, Ospina E, Basso K, Nandula S, Murty V, Baer R, Ludwig T. The basal-like mammary carcinomas induced by Brca1 or Bard1 inactivation implicate the BRCA1/BARD1 heterodimer in tumor suppression. Proc Natl Acad Sci USA 2008; 105: 7040-7045.

28. Smid M, Wang YX, Zhang Y, Sieuwerts AM, Yu J, Klijn JGM, Foekens JA, Martens JWM. Subtypes of breast cancer show preferential site of relapse. Cancer Res 2008; 68: 3108-3114.

29. Van den Eynden GG, Smid M, Van Laere SJ, Colpaert CG, Van der Auwera I, Bich TX, van Dam P, den Bakker MA, Dirix LY, Van Marck EA, Vermeulen PB, Foekens JA. Gene expression profiles associated with the presence of a fibrotic focus and the growth pattern in lymph node-negative breast cancer. Clin Cancer Res 2008; 14: 2944-2952.

30. Vultur A, Buettner R, Kowolik C, Liang W, Smith D, Boschelli F, Jove R. SKI-606 (bosutinib), a novel Src kinase inhibitor, suppresses migration and invasion of human breast cancer cells. Mol Cancer Ther 2008; 7: 1185-1194.

31. Wu KM, Katiyar S, Li AP, Liu MR, Ju XM, Popov VM, Jiao XM, Lisanti MP, Casola A, Pestell RG. Dachshund inhibits oncogeneinduced breast cancer cellular migration and invasion through suppression of interleukin-8. Proc Natl Acad Sci USA 2008; 105: 6924-6929.

32. Yang XWH, Richardson AL, Torres-Arzayus MI, Zhou PC, Sharma C, Kazarov AR, Andzelm MM, Strominger JL, Brown M, Hemler ME. CD151 accelerates breast cancer by regulating $\alpha(6)$ integrin function, signaling, and molecular organization. Cancer Res 2008; 68: 3204-3213.

Prepared by $R$ Sutherland Cancer Research Program Garvan Institute of Medical Research Darlinghurst, NSW, Australia 